Research Article

\title{
A Synthetic Pan-Aurora Kinase Inhibitor, 5-Methoxy-2-(2-methoxynaphthalen-1-yl)-4H-chromen-4-one, Triggers Reactive Oxygen Species-Mediated Apoptosis in HCT116 Colon Cancer Cells
}

\author{
Jeong Yeon Lee $\mathbb{D}^{1},{ }^{1}$ Sung Shin Ahn $\mathbb{D}^{1},{ }^{1}$ You Jeong Jeong $\mathbb{D},{ }^{1}$ Jihye Choi $\mathbb{D},{ }^{1}$ \\ Seunghyun Ahn $\mathbb{D}^{2},{ }^{2}$ Dongsoo Koh $\mathbb{D}^{2},{ }^{2}$ Young Han Lee $\mathbb{D},{ }^{1,3}$ Yoongho Lim $\mathbb{D}$, ${ }^{3,4}$ \\ and Soon Young Shin $\mathbb{D}^{1,3}$ \\ ${ }^{1}$ Department of Biological Sciences, Sanghuh College of Life Sciences, Konkuk University, Seoul 05029, Republic of Korea \\ ${ }^{2}$ Department of Applied Chemistry, Dongduk Women's University, Seoul 02748, Republic of Korea \\ ${ }^{3}$ Cancer and Metabolism Institute, Konkuk University, Seoul 05029, Republic of Korea \\ ${ }^{4}$ Division of Bioscience and Biotechnology, BMIC, Konkuk University, Seoul 05029, Republic of Korea \\ Correspondence should be addressed to Soon Young Shin; shinsy@konkuk.ac.kr
}

Received 19 March 2020; Accepted 13 May 2020; Published 2 July 2020

Academic Editor: Thomas R. Caulfield

Copyright (C) 2020 Jeong Yeon Lee et al. This is an open access article distributed under the Creative Commons Attribution License, which permits unrestricted use, distribution, and reproduction in any medium, provided the original work is properly cited.

\begin{abstract}
Aurora kinases are Ser/Thr kinases that function as mitotic regulators. 5-Methoxy-2-(2-methoxynaphthalen-1-yl)-4H-chromen-4-one (DK1913) is a synthetic pan-Aurora kinase inhibitor. However, the mode of action of DK1913 concerning the induction of apoptosis is unclear. Here, we report that DK1913 triggered apoptosis, as revealed by flow cytometry and Annexin V staining. DK1913 enhanced the intracellular levels of reactive oxygen species (ROS) and stimulated the endoplasmic reticulum (ER) and genotoxic stress responses. We also found that DK1913 induced the loss of mitochondrial membrane potential, leading to the activation of caspase- 9 , caspase-7, and caspase-3. In addition, the antioxidant, butylated hydroxyanisole (BHA), abrogated DK1913-induced loss of mitochondrial membrane potential and activation of the caspase cascade. These findings demonstrate that pan-Aurora kinase inhibitor DK1913 triggers apoptosis through ROSmediated ER and genotoxic stress responses.
\end{abstract}

\section{Introduction}

Cell division is an essential event for normal growth and development. One of the primary purposes of mitosis is the correct segregation of the duplicated chromosomes to two daughter cells. Mitotic progression is regulated by a complex network of mitotic kinases [1]. The aurora kinase family members (Aurora-A, Aurora-B, and Aurora-C) are Ser/Thr protein kinases that play critical roles in the regulation of mitotic progression [2]. The subcellular distribution and functions of the aurora kinases are largely distinct; Aurora-A is a mitotic centrosomal kinase required for centrosomal maturation, centrosomal microtubule organization, and bipolar spindle assembly during mitosis [3], Aurora-B is present on the mitotic spindle and plays a role in chromosome condensation, sister chromatid cohesion, mitotic spindle assembly, and chromosomal alignment [2], and Aurora-C is localized to the centrosome and plays a role in centrosome function at a later stage of mitosis [4]. Aurora kinases are overexpressed in many human cancers, including colon cancer [5], and deregulation of their kinase activity can lead to chromosomal instability, which results in tumorigenesis [6]. Inhibition of aurora kinases induces mitotic cell cycle arrest and eventually triggers apoptotic cell death [7]. Therefore, targeting Aurora kinases is a valuable strategy when designing novel drugs in cancer therapy.

In a previous study, we investigated the structural features of flavone derivatives that inhibit the aurora kinase activity [8]. In that study, we found that 5-methoxy-2-(2methoxynaphthalen-1-yl)-4H-chromen-4-one

(DK1913) 
directly binds to Aurora-A and Aurora-B and induces cell cycle arrest at the G2/M phase, eventually leading to apoptotic cell death. As the aurora kinases are associated with mitotic signaling, it seems clear that inhibition of the aurora kinase could be linked to the cell cycle arrest at the G2/M phase. However, the molecular mechanisms responsible for the induction of apoptosis remain elusive.

The mitotic arrest can increase the intracellular level of reactive oxygen species (ROS), thereby inducing oxidative stress [9]. ROS comprises oxygen-containing reactive molecules, such as superoxide anion radical $\left(\mathrm{O}_{2}{ }^{-}\right)$, hydrogen peroxide $\left(\mathrm{H}_{2} \mathrm{O}_{2}\right)$, hydroxyl radical ( $\left.\mathrm{OH}\right)$, and singlet oxygen $\left({ }^{1} \mathrm{O}_{2}\right)$. Aberrant ROS production can lead to cellular damages known as oxidative stress. Although oxidative stress has been implicated in various human diseases, there is increasing evidence regarding the beneficial effects of ROS with respect to the selective killing of cancer cells $[10,11]$. Aberrant accumulation of ROS can activate the unfolded protein response (UPR) in the endoplasmic reticulum (ER). Prolonged UPR has been implicated in the induction of ER stress, resulting in apoptotic cell death $[12,13]$.

In this study, we investigated whether DK1913 induces apoptosis through ROS generation in HCT116 colon cancer cells. We found that DK1913-induced apoptosis is associated with ROS-mediated ER and genotoxic stress responses. In addition, we have shown that DK1913 induces the loss of mitochondrial membrane potential $(\Delta \Psi \mathrm{m})$, which leads to the activation of the caspase cascade.

\section{Materials and Methods}

2.1. Materials. DK1913 compound (Figure 1(a)) was synthesized as described previously [8]. Primary antibodies against phospho-Aurora-A (T288)/Aurora-B (T232)/Aurora-C (T198), cleaved caspase-7 (Asp198), and poly (ADPribose) polymerase (PARP), $\gamma$-H2AX (S139), caspase-2, caspase-9, cleaved caspase-7, and caspase- 3 were obtained from Cell Signaling Technology (Beverly, MA, USA). Primary antibodies specific to p53, BID, BIM, BAX, and GAPDH were obtained from Santa Cruz Biotechnology (Santa Cruz, CA, USA). Fluorescein isothiocyanate- (FITC-) conjugated Annexin V kit and anti-Cyt $c$ antibody were purchased from BD Pharmingen (San Diego, CA, USA). Alexa Fluor ${ }^{\circledR} 488$ AffiniPure F $\left(\mathrm{ab}^{\prime}\right)_{2}$ Fragment Goat AntiMouse IgG $(\mathrm{H}+\mathrm{L})$ was procured from Jackson ImmunoResearch Inc. (West Grove, PA, USA). DCF-DA, BHA, and Hoechst 33258 were obtained from Sigma-Aldrich (St. Louis, MO, USA). JC-1 mitochondrial membrane potential assay kit was obtained from Cayman Chemical (Ann Arbor, MI, USA). MitoTracker Red CMXRos dye (catalog \# M-7512) was procured from Invitrogen (Waltham, MA, USA). Other chemicals were obtained from Sigma-Aldrich.

2.2. Cell Culture. HCT116 human colon cancer cells were obtained from the American Type Culture Collection (Rockville, MD, USA). The cells were maintained in Dulbecco's modified Eagle's medium supplemented with 10\% fetal bovine serum (CellGro/Corning, Manassas, VA, USA) at $37^{\circ} \mathrm{C}$ in a $5 \% \mathrm{CO}_{2}$ atmosphere, as described previously [14].

2.3. Cell Viability. Cell viability was determined using a water-soluble colorimetric Cell Counting Kit-8 (CCK-8; Dojindo Molecular Technologies, Gaithersburg, MD, USA), based on the reduction of slightly yellow color 2-(2methoxy-4-nitrophenyl)-3-(4-nitrophenyl)-5-(2,4-disulfophenyl)-2H-tetrazolium) to orange color formazan product by intracellular dehydrogenase activities. HCT116 cells $\left(1 \times 10^{3}\right.$ cells/plate $)$ were treated with increasing concentrations of DK1913 $(0-20 \mu \mathrm{M})$ for $24 \mathrm{~h}$. After adding the CCK- 8 solution, cells were incubated at $37^{\circ} \mathrm{C}$ and $5 \%$ $\mathrm{CO}_{2}$ for $2 \mathrm{~h}$. Absorbance at $450 \mathrm{~nm}$ was measured using an Emax Endpoint ELISA Microplate Reader (Molecular Devices, Sunnyvale, CA, USA).

2.4. Immunoblot Analysis. HCT116 cells were extracted in lysis buffer containing $20 \mathrm{mM}$ HEPES ( $\mathrm{pH} 7.2$ ), 1\% Triton $\mathrm{X}-100,10 \%$ glycerol, $150 \mathrm{mM} \mathrm{NaCl}, 10 \mu \mathrm{g} / \mathrm{mL}$ leupeptin, and $1 \mathrm{mM}$ phenylmethylsulfonyl fluoride (PMSF). The protein extracts $(20 \mu \mathrm{g}$ each) were electrophoresed on a $10 \%$ SDSpolyacrylamide gel and transferred onto a nitrocellulose membrane. The blots were incubated with the appropriate primary and secondary antibodies, and antibody-reactive proteins were visualized using an enhanced chemiluminescence detection system (GE Healthcare, Piscataway, NJ, USA). Relative band intensities were analyzed using the ImageJ software version $1.52 \mathrm{a}$ (National Institute of Health, Bethesda, MD, USA) and expressed relative to the basal control band (set to 1) after normalizing with GAPDH levels.

2.5. Cell Cycle Analysis by Flow Cytometry. Cell cycle distribution was examined by flow cytometry, as described previously [15]. Briefly, HCT116 cells were treated with $5 \mu \mathrm{M}$ DK1913 for $24 \mathrm{~h}$ and then fixed in $70 \%(v / v)$ ethanol, followed by staining with $50 \mu \mathrm{g} / \mathrm{mL}$ propidium iodide (PI) solution containing $0.1 \%(v / v)$ Triton X-100, $0.1 \mathrm{mM}$ EDTA, and $50 \mu \mathrm{g} / \mathrm{mL}$ RNase A. The PI fluorescence was measured by a NucleoCounter NC-3000 cytometer (ChemoMetec, Allerød, Denmark). Diploid (2N) and tetraploid (4N) cells containing between $2 \mathrm{~N}$ and $4 \mathrm{~N}$ DNA were considered to be in the G1, G2/M, and S phases, respectively. Cells with less than $2 \mathrm{~N}$ DNA (sub-G1 phase) were regarded as apoptotic cells [16].

2.6. Apoptosis Assay by Annexin V Staining. Apoptosis assay was performed using a FITC-conjugated Annexin V kit according to the manufacturer's instructions (BD Pharmingen). Fluorescence was measured using a NucleoCounter NC-3000 image cytometer (ChemoMetec), as described previously [17].

2.7. Detection of Intracellular Reactive Oxygen Species (ROS). Intracellular levels of ROS were determined using oxidantsensing fluorescent probe DCF-DA. After treatment with 


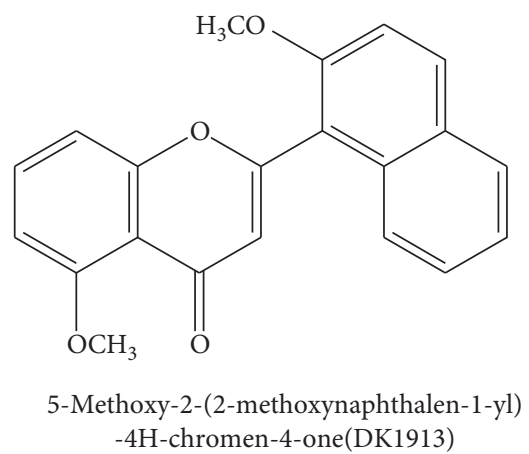

(a)
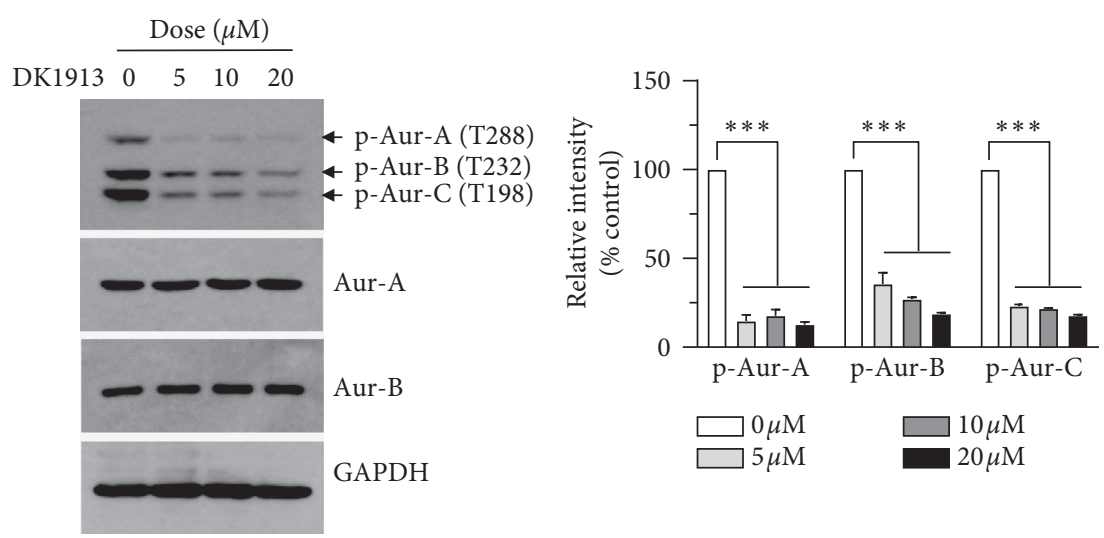

(b)

FIGURE 1: Effects of DK1913 on the inhibition of aurora kinases. (a) Chemical structure of DK1913. (b) HCT116 cells were treated with different concentrations of DK1913 $(0,5,10$, and $20 \mu \mathrm{M})$ for $30 \mathrm{~min}$. Total cell lysates were immunoblotted with a phospho-specific antibody, which can detect phospho-Aurora-A (T288)/Aurora-B (T232)/Aurora-C (T198). Band intensities of phosphorylated Aurora-A, Aurora- $\mathrm{B}$, and Aurora- $\mathrm{C}$ were measured using ImageJ software and expressed relative to the basal control bands (set to $100 \%)$ after normalizing with total proteins (Aur-A and Aur-B) or GAPDH (Aur-C) levels. Aur, Aurora; bars, means $\pm \mathrm{SD}(n=3) .{ }^{* * *} P<0.001$ by Sidak's multiple comparisons test.

DK1913, the cells were incubated with $10 \mu \mathrm{M}$ DCF-DA for an additional $30 \mathrm{~min}$. DCF fluorescence resulting from ROS production was measured using a FACSCalibur flow cytometer (BD Biosciences, San Jose, CA, USA). Fluorescent intracellular ROS were also visualized by an EVOSf1 fluorescence microscope (Advance Microscopy Group, Bothell, WA, USA).

\subsection{Measurement of the Mitochondrial Membrane Potential} $(\Delta \Psi m)$. Loss of the mitochondrial membrane potential was monitored by measuring the fluorescence of the lipophilic cationic probe JC-1 (5,5',6,6'-tetrachloro $1,1^{\prime}, 3,3^{\prime}$-tetraethylbenzimi-dazolylcarbocyanine chloride), according to the manufacturer's instruction (Cayman Chemical). HCT116 cells were seeded into a 24 -well plate and treated with $5 \mu \mathrm{M}$ DK1913. After $18 \mathrm{~h}$, the cells were treated with JC-1 dye and incubated for an additional $30 \mathrm{~min}$ at $37^{\circ} \mathrm{C}$, according to the manufacturer's instructions (Cayman Chemical). Fluorescent images were captured under an EVOSf $1{ }^{\circledR}$ fluorescence microscope. The aggregated JC-1 in mitochondria indicates high membrane potential and emits red fluorescence, while monomeric JC-1 indicates loss of membrane potential and emits green fluorescence.

2.9. Statistical Analysis. Statistical significance was analyzed using GraphPad PRISM software version 8.2.1 (GraphPad Software Inc., La Jolla, CA). A $P$ value of less than 0.05 was considered significant. All experiments were performed in triplicate.

\section{Results and Discussion}

3.1. DK1913 Inhibits the Aurora Kinases in HCT116 Colon Cancer Cells. To investigate the mode of action of DK1913 (Figure 1(a)) concerning the induction of apoptosis, we first confirmed the inhibitory effect of DK1913 on the phosphorylation of Aurora kinases by immunoblot analysis. Treatment with DK1913 significantly $(P<0.001, n=3)$ prevented phosphorylation of Aurora-A, Aurora-B, and Aurora-C at a concentration of less than $5 \mu \mathrm{M}$ (Figure 1(b)).

3.2. DK1913 Induces G2/M Cell Cycle Arrest and Apoptosis. To determine the cytotoxicity of DK1913, we performed a cell viability assay using a CCK-8. The cell viability was $95.7 \pm 4.16 \%, 82.7 \pm 8.62 \%, 73.3 \pm 7.09 \%, 61.7 \pm 4.51 \%$, and $36.7 \pm 11.2 \%$ at $1,5,10,20$, and $40 \mu \mathrm{M}$, respectively, as compared with the vehicle-treated control $(0 \mu \mathrm{M})$. These results suggest that DK1913 reduced cell viability in a dosedependent fashion (Figure 2(a)). We next tested the effect of DK1913 on cell cycle and apoptosis by flow cytometry. As expected, DK1913 increased the population of G2/M phase cells from $11.9 \%$ to $58.3 \%$ with a concomitant decrease in G1 and an increase in sub-G1 cell population (Figure 2(b)). Translocation of phosphatidylserine (PS) from the inner layer to the outer leaflet is a typical marker of apoptosis [18]. We thus examined the effect of DK1913 on the localization of PS on the membrane surface using fluorescein-5-isothiocyanate (FITC)-conjugated Annexin V (AV). Propidium iodide (PI) was used to detect necrotic cells. Treatment with DK1913 increased the population of AV-positive cells from $9 \%$ to $97 \%$ (Figure 2(c)). These results demonstrate that DK1913 induces cell cycle arrest at the G2/M phase and triggers apoptotic cell death in HCT116 colon cancer cells.

3.3. DK1913 Generates Intracellular Reactive Oxygen Species (ROS). Several reports have demonstrated that inhibition of the aurora kinase can induce G2/M arrest and apoptosis in various cancer cells $[19,20]$. However, very little is understood about how DK1913 causes apoptosis. Many polyphenols, such as quercetin and resveratrol, produce intracellular ROS to induce apoptosis in various cancer cells 


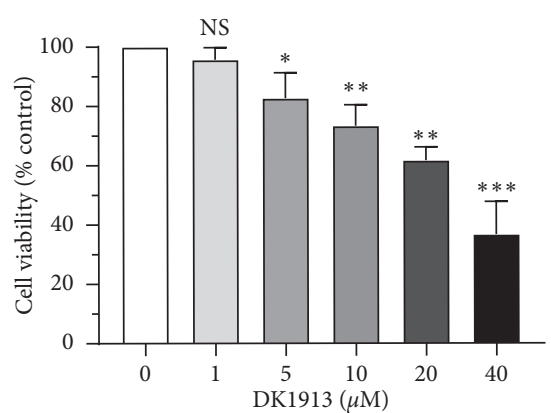

(a)

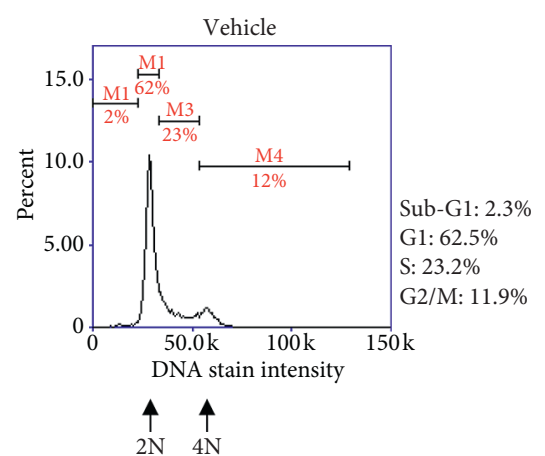

$\begin{array}{cc}4 & 4 \\ 2 \mathrm{~N} & 4 \mathrm{~N}\end{array}$

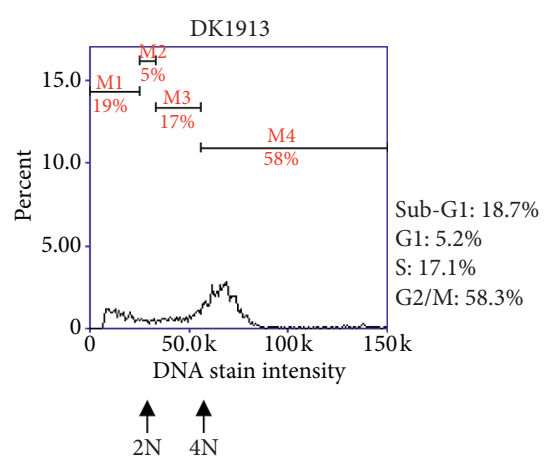

(b)
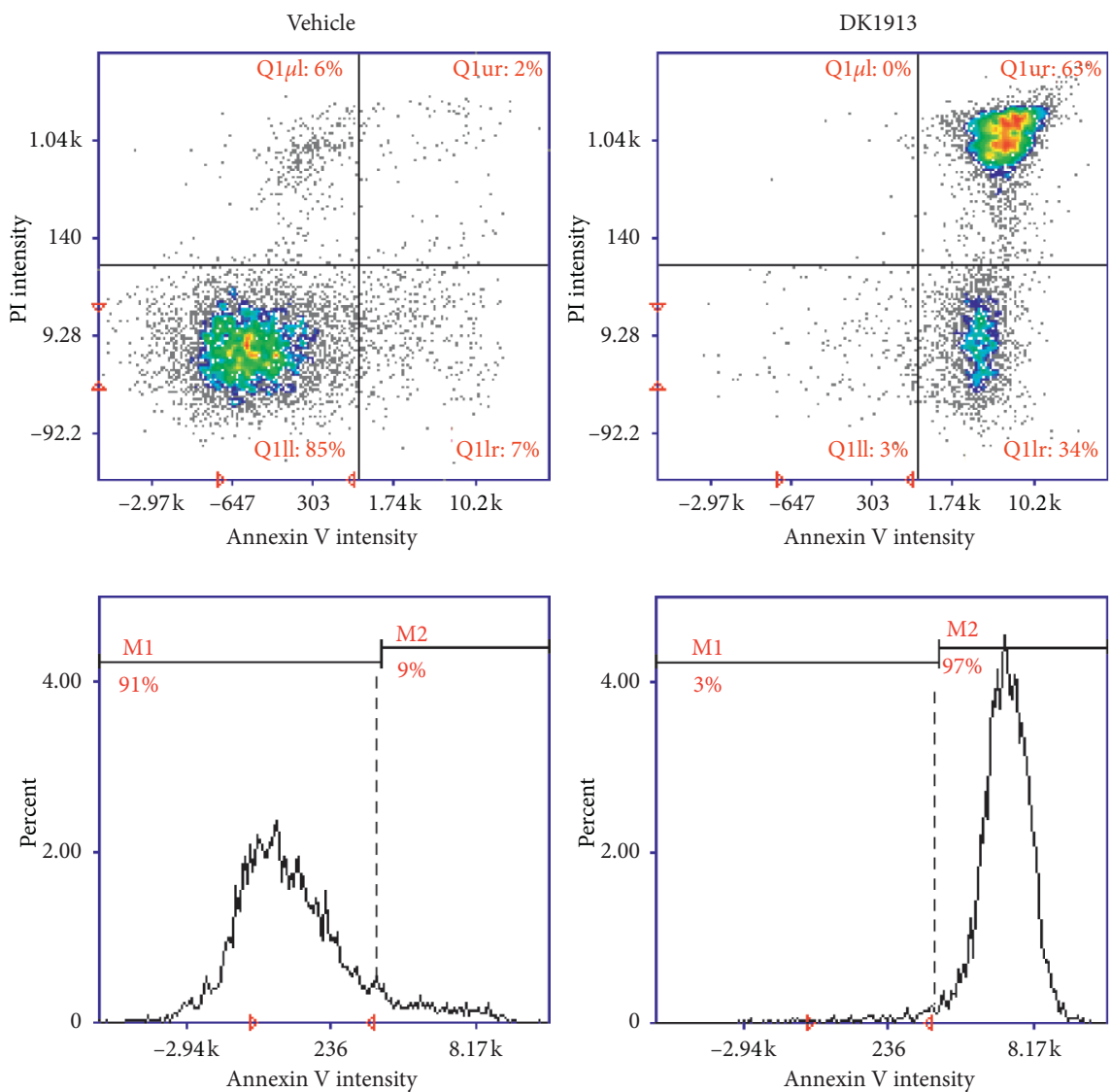

(c)

FIGURE 2: Effect of DK1913 on the induction of cell cycle arrest and apoptosis. (a) HCT116 cells $\left(1 \times 10^{3}\right.$ cells/sample) were treated with different concentrations of DK1913 for $24 \mathrm{~h}$. Cell viability was measured using a cell counting kit- 8 . Data are presented as means \pm SD $(n=3)$. NS, not significant $(P=0.900) ;{ }^{*} P=0.037 ;{ }^{* *} P<0.01 ;{ }^{* * *} P<0.001$ by Dunnett's multiple comparisons test. (b) HCT116 cells $\left(3 \times 10^{5}\right.$ cells/sample) were treated with either vehicle (dimethyl sulfoxide, DMSO) or $5 \mu \mathrm{M}$ DK1913 for $24 \mathrm{~h}$. After staining with propidium iodide (PI), cellular DNA contents were measured with a NucleoCounter NC-3000 cytometer. M1, sub-G1 phase; M2, G1 phase; M3, S phase; M4, G2/M phase. 2N: diploid DNA content; 4N: tetraploid DNA content. (c) HCT116 cells were treated with $5 \mu \mathrm{M}$ DK1913 for $48 \mathrm{~h}$ and then stained with propidium iodide (PI) and FITC-Annexin V. Fluorescence intensities were analyzed using a NucleoCounter NC-3000 cytometer. Scatter plots (top panels) show FITC-Annexin V intensity ( $x$-axis) versus PI intensity ( $y$-axis). Histograms (bottom panels) show FITC-Annexin V intensity ( $x$-axis) versus the percentage of the cell population ( $y$-axis). M1, Annexin V negative; M2, Annexin V positive.

$[21,22]$. As aberrant ROS production in cancer cells may increase the efficacy of chemotherapeutic anticancer agents $[23,24]$, we investigated whether DK1913 elevated the levels of intracellular ROS. We detected ROS using the redoxsensitive $2^{\prime}, 7^{\prime}$-dichlorofluorescein diacetate (DCF-DA) dye. Flow cytometry showed that DK1913 increased DCF fluorescence within $6 \mathrm{~h}$, which remained elevated relative to the control for more than $12 \mathrm{~h}$ (Figure 3(a)). To further assess ROS generation by DK1913, we visualized intracellular ROS using DCF-DA as a fluorescent probe in cultured cells with fluorescence microscopy. There was no noticeable fluorescence in vehicle-treated cells; however, after $12 \mathrm{~h}$ of 

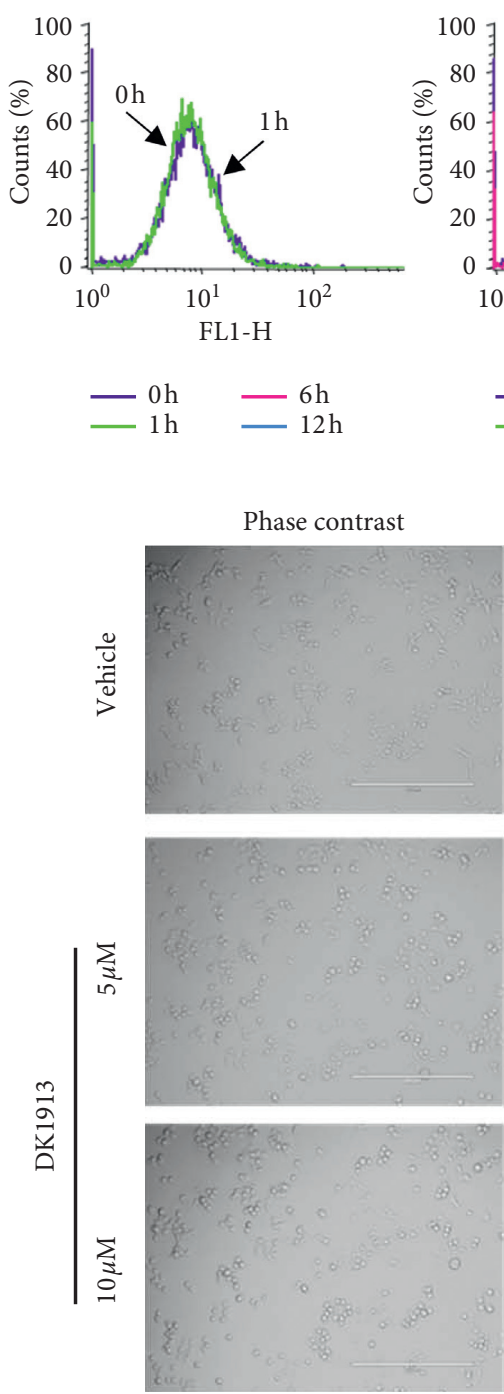
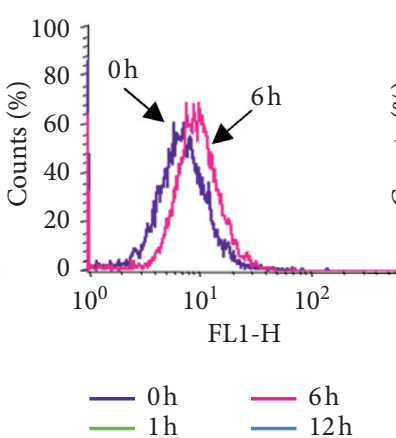
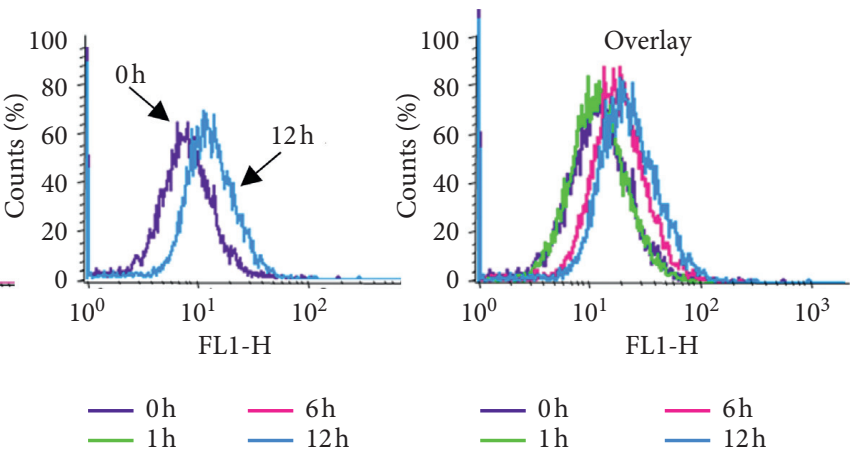

(a)
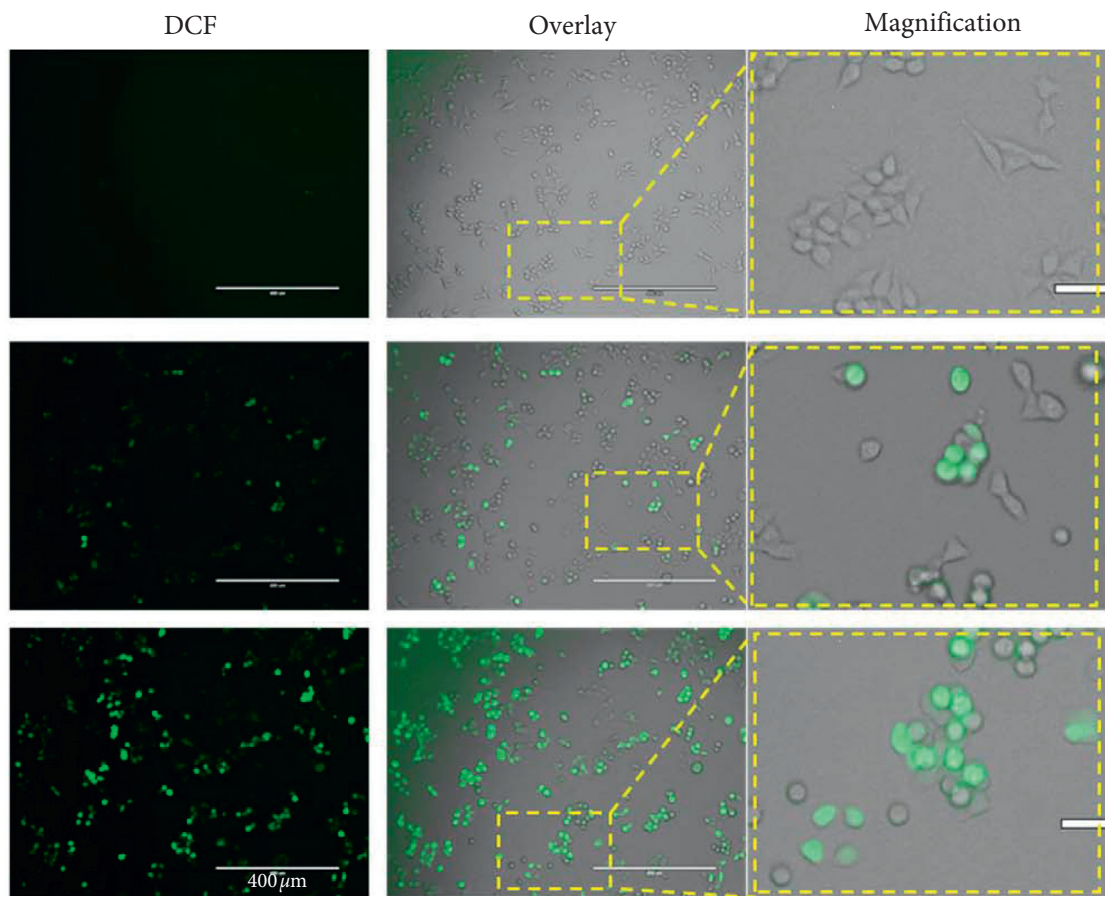

(b)

FIGURE 3: Effect of DK1913 on the generation of reactive oxygen species (ROS) in HCT116 cells. (a) HCT116 cells were incubated with $10 \mu \mathrm{M}$ DCF-DA for $60 \mathrm{~min}$, followed by incubation with $5 \mu \mathrm{M}$ DK1913 for different periods $(0,1,6$, and $12 \mathrm{~h})$. DCF fluorescence was assessed using flow cytometry performed on a FACSCalibur flow cytometer. (b) HCT116 cells were incubated with $10 \mu \mathrm{M}$ DCF-DA for 60 min, followed by incubation with $5 \mu \mathrm{M}$ DK1913 for $12 \mathrm{~h}$. Intracellular ROS were visualized using an EVOSf1 fluorescence microscope (scale bar, $400 \mu \mathrm{m}$ ).

DK1913 treatment, the number of fluorescent cells increased dose-dependently (Figure 3(b)). These data suggest that DK1913 generates intracellular ROS.

3.4. DK1913 Induces the Endoplasmic Reticulum (ER) Stress. ROS can induce apoptosis through unfolded protein response- (UPR-) mediated ER stress response [25]. A typical feature of the UPR is the accumulation of ER-resident UPR sensor proteins. To determine whether DK1913 stimulates the UPR pathway, we investigated the effect of DK1913 on the expression of ER-resident UPR sensor proteins. As shown in Figure 4, DK1913 increased the amounts of ER stress sensor (GRP78), ER stress effectors (PERK and IRE $1 \alpha$ ), and transcription factors ATF4, and spliced form of XBP1 (sXBP1) in a time-dependent manner, suggesting the activation of the ER stress response by DK1913. Under sustained ER stress conditions, ATF4 and sXBP1 contributed to the induction of apoptosis by upregulating the proapoptotic transcription factor C/EBP homologous protein (CHOP), also known as growth arrest- and DNA damage-inducible gene 153 (GADD153) [26]. We also found that DK1913 increased the amounts of CHOP and proapoptotic BCL2-interacting protein (BIM), a downstream target of CHOP [27], $12 \mathrm{~h}$ after treatment. These data suggest that DK1913 causes the activation of the UPR-mediated ER stress.

3.5. DK1913 Induces the Genotoxic Response. Tumor suppressor p53 plays a central role in cellular genotoxic stressinduced apoptosis [28]. Prolonged ER stress can upregulate p53 expression. To test whether p53 plays a role in DK1913induced apoptosis, we examined the expression levels of p53. 


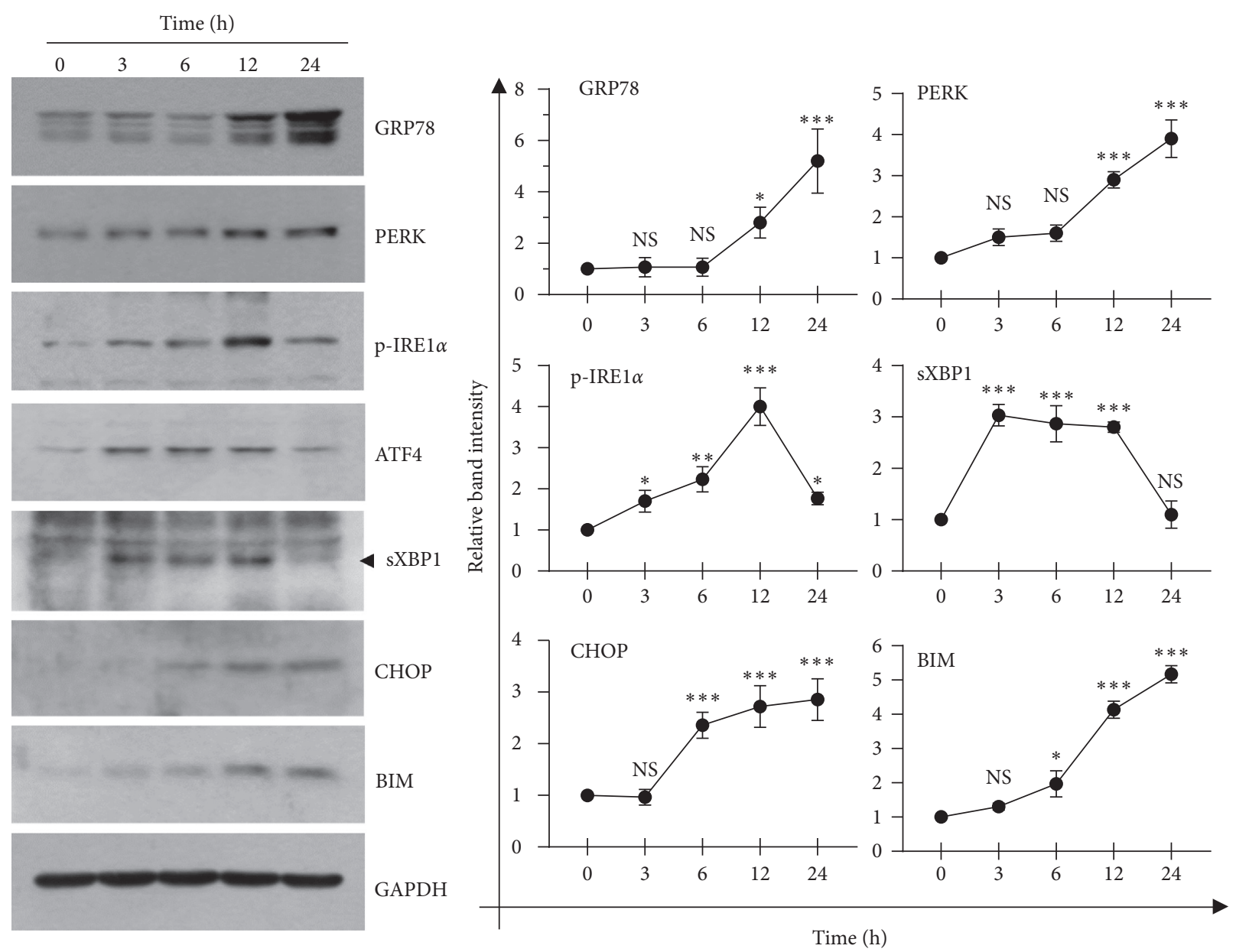

FIGURE 4: Effect of DK1913 on the activation of the unfolded protein response (UPR) pathway in HCT116 cells. HCT116 cells were treated with $5 \mu \mathrm{M}$ DK1913 for different time periods. Cell lysates were subjected to immunoblotting using antibodies against ER stress response proteins. Arrowhead: spliced form of XBP1 (sXBP1); GAPDH: internal control. Band intensities of each blot were measured using ImageJ software and expressed relative to the basal control band (set to 1) after normalization with GAPDH levels. The data are expressed as means \pm SD $(n=3)$. NS, not significant; ${ }^{*} P<0.05 ;{ }^{* *} P<0.01$; ${ }^{* * *} P<0.001$; compared with the 0 -time control according to Dunnett's multiple comparisons test.

As shown in Figure 5, the amounts of phosphorylated p53 at Ser-15 were significantly increased after $6 \mathrm{~h}$ of DK1913 treatment. BCL2-associated X protein (BAX), a downstream target of p53 [29], was significantly increased after $12 \mathrm{~h}$. Phosphorylation of histone variant H2AX at Ser-139 (termed $\gamma$-H2AX), a hallmark of DNA damage [30], was significantly increased $24 \mathrm{~h}$ after DK1913 treatment. These results suggest the induction of genotoxic stress by DK1913. Caspase-2, a cysteine protease that is a member of the interleukin- $\beta \beta$-converting enzyme (ICE) family, mediates DNA damage-induced apoptosis by producing a truncated form of BID (t-BID) [31, 32]. DK1913 also increased the proteolytic cleavage of pro-caspase- 2 and t-BID in a timedependent manner. These data suggest that DK1913 induces a genotoxic stress response.

3.6. DK1913 Induces Activation of the Caspase Cascade. Mitochondria play a pivotal role in triggering the intrinsic apoptosis pathway. Caspases are cysteine-dependent aspartate-specific proteases that mediate apoptotic signaling [33].
Oligomerization of proapoptotic BAX results in the formation of a membrane channel and facilitates the release of Cyt $\mathrm{c}$ from the mitochondria into the cytosol [34]. BCL2 homology domain 3- (BH3-) containing proteins, including BIM and $t$-BID, stimulate the release of Cyt $c$ through inhibition of BCL2 [35]. The Cyt $c$ released in the cytosol binds to APAF1 and pro-caspase- 9 to form a complex called an apoptosome. Within the apoptosome, pro-caspase- 9 becomes activated, leading to activation of the executioner caspases (caspase-3 and caspase-7), which triggers apoptosis [36].

As DK1913 increased the levels of BAX, BIM, and $t$-BID, we sought to determine the activation status of caspases by immunoblotting (Figure 6). We found that the proteolytic processing of the initiator caspase (caspase-9) and executioner caspases (caspase- 3 and caspase-7), as well as the cleavage of poly (ADP-ribose) polymerase (PARP), a hallmark of the activation of executioner caspases [37], was increased following DK1913 treatment. These data suggest that DK1913 triggers the intrinsic apoptotic pathway through activation of the caspase cascade. 


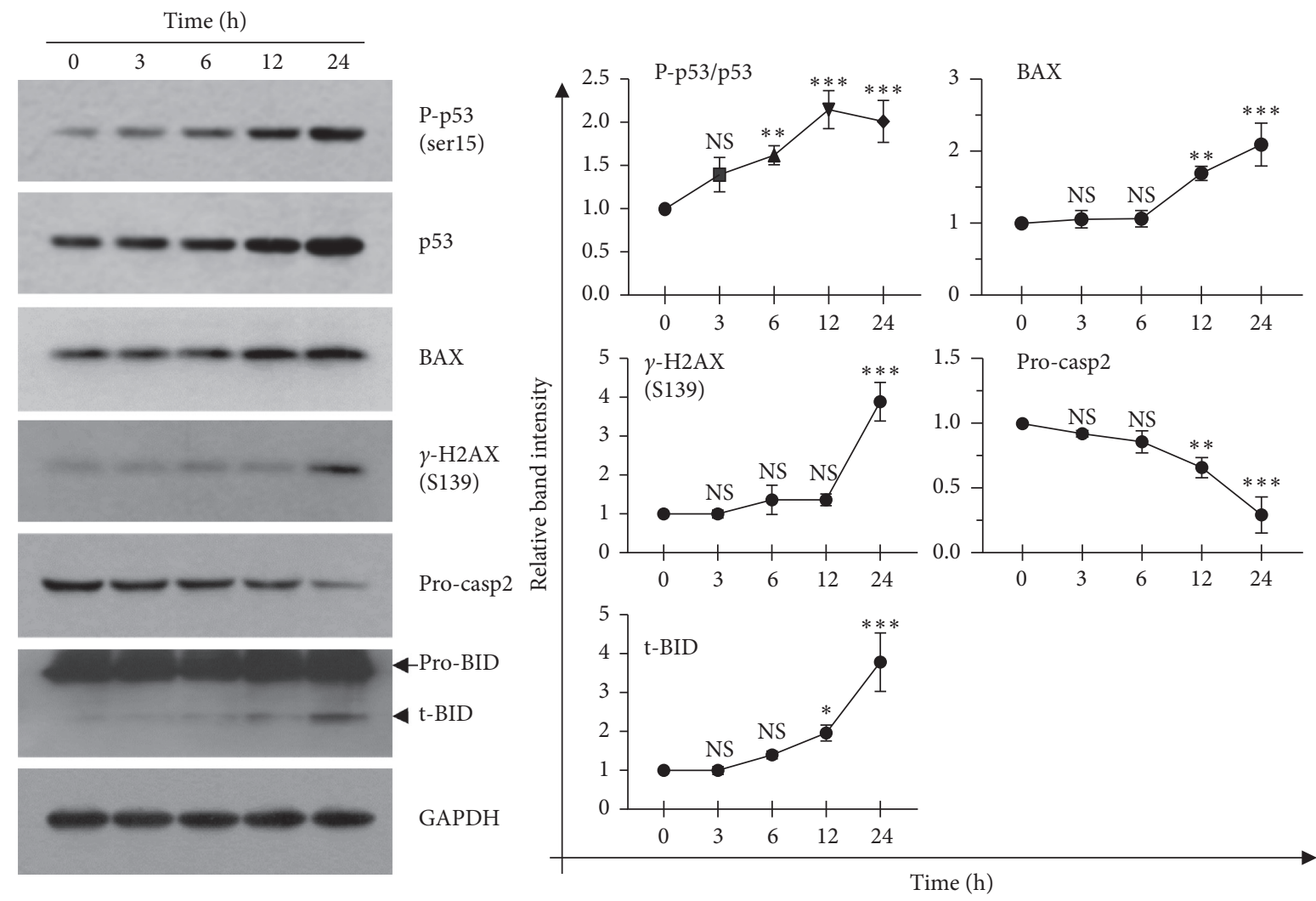

FIGURE 5: Effect of DK1913 on the induction of genotoxic stress response in HCT116 cells. HCT116 cells were treated with $5 \mu \mathrm{M}$ DK1913 for different time periods. Cell lysates were subjected to immunoblotting using antibodies against genotoxic response proteins. Arrow: proform of BID (pro-BID); arrowhead: truncated form of BID (t-BID); GAPDH: internal control. Band intensities in each blot were measured using ImageJ software and expressed relative to the basal control band (set to 1 ) after normalizing with p53 or GAPDH. The data are presented as means \pm SD ( $n=3$ ). NS, not significant; * $P<0.05 ;{ }^{* *} P<0.01$; ${ }^{* * *} P<0.001$; compared with the 0 -time control according to Dunnett's multiple comparisons test.
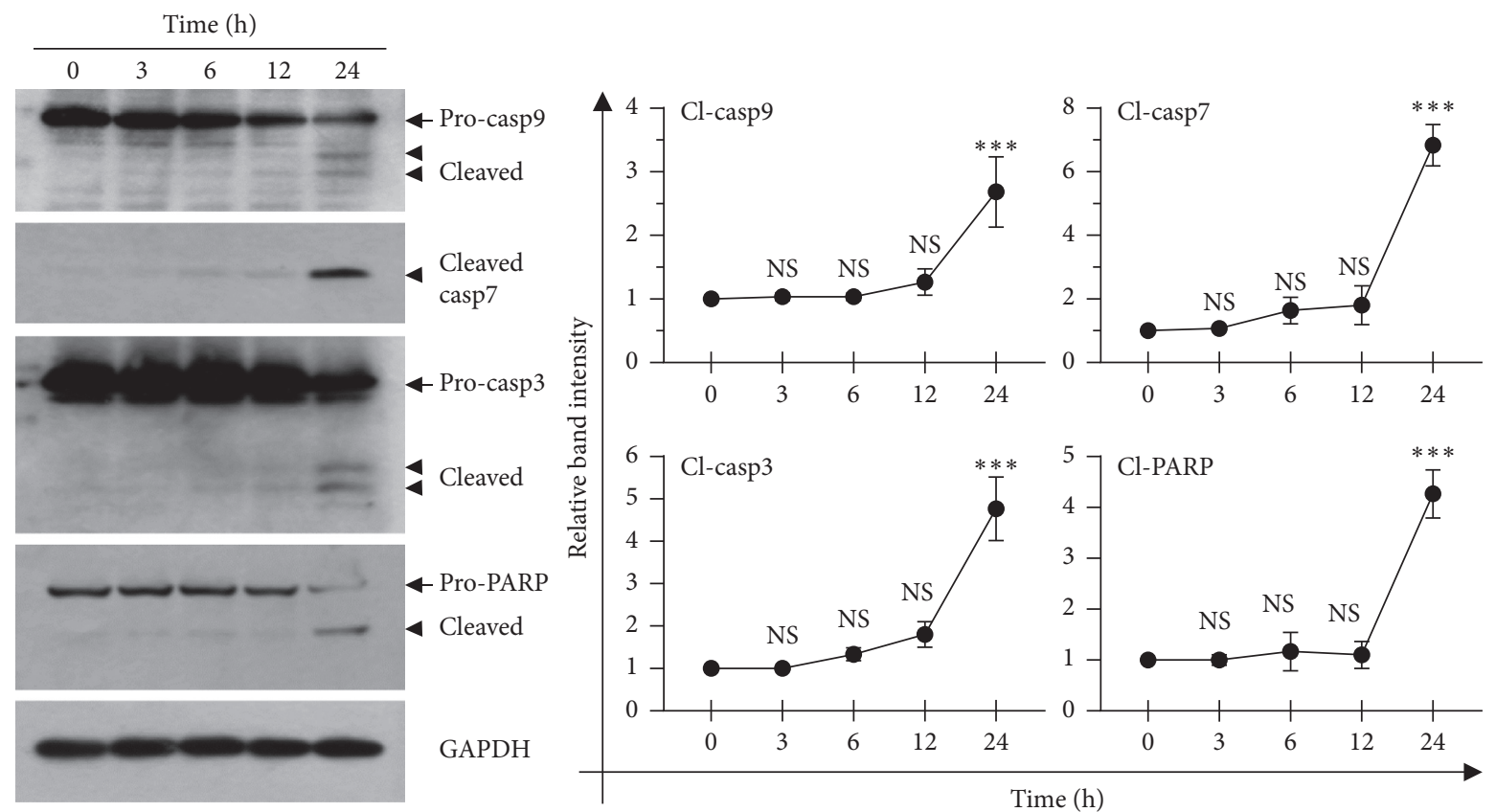

FIGURE 6: Effect of DK1913 on the activation of mitochondria-mediated caspase cascade in HCT116 cells. HCT116 cells were treated with $10 \mu \mathrm{M}$ DK1913 for $24 \mathrm{~h}$. Cell lysates were subjected to immunoblotting using antibodies against caspase-9, cleaved caspase-7, caspase-3, and PARP. Arrows: proform; arrowhead: cleaved form; GAPDH: internal control. Band intensities of each blot were measured using ImageJ software and expressed relative to the basal band intensity (set to 1$)$ after adjusting to GAPDH levels. The data are expressed as means \pm SD $(n=3)$. NS, not significant; ${ }^{*} P<0.05 ;{ }^{* *} P<0.01 ;{ }^{* * *} P<0.001$; compared with the 0 -time control according to Dunnett's multiple comparisons test. 

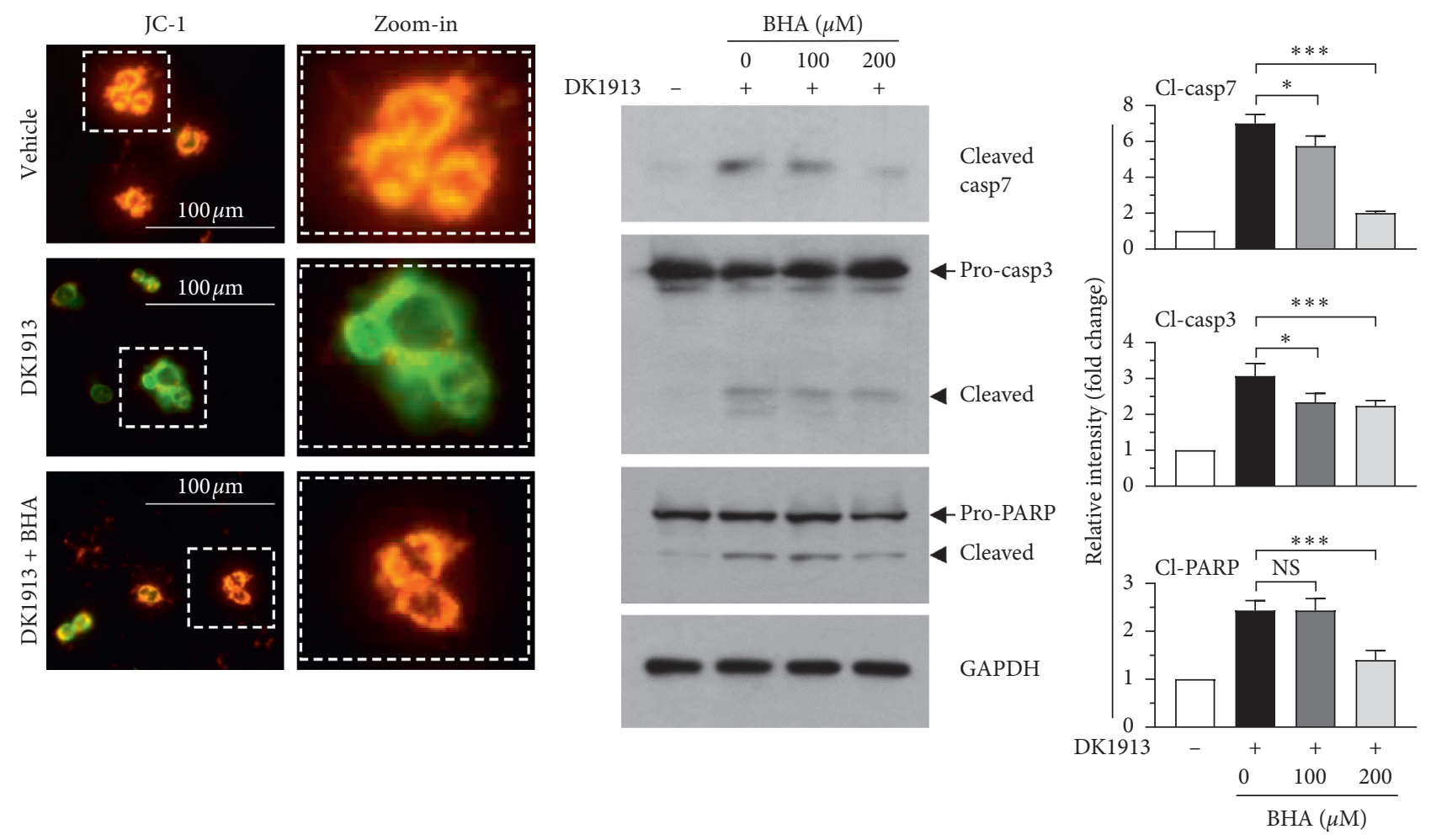

(a)

(b)

FIGURE 7: Effect of antioxidants on the inhibition of DK1913-induced loss of mitochondria membrane potential $(\Delta \Psi \mathrm{m})$ and activation of the caspase cascade. (a) HCT116 cells were pretreated with $100 \mu \mathrm{M}$ butylated hydroxyanisole (BHA) for 30 min before the addition of $5 \mu \mathrm{M}$ DK1913. After $18 \mathrm{~h}$, the $\Delta \Psi \mathrm{m}$-sensitive dye JC-1 was incubated for an additional $10 \mathrm{~min}$. Red and green fluorescence was visualized by an EVOSf1 ${ }^{\circledR}$ fluorescence microscope (scale bar, $\left.100 \mu \mathrm{m}\right)$. (b) HCT116 cells were treated with different concentrations of BHA $(0,100$, and $200 \mu \mathrm{M}$ ) for $30 \mathrm{~min}$, followed by treatment with $5 \mu \mathrm{M}$ DK1913. After $24 \mathrm{~h}$, cell lysates were prepared and immunoblotting was performed using antibodies against cleaved caspase-7, caspase-3, and PARP. Arrow: proform; arrowhead: cleaved form; GAPDH: internal control. Band intensities of each blot were measured using ImageJ software and expressed relative to the basal band intensity (ser to 1) after adjusting to GAPDH levels. The data are presented as means $\pm \mathrm{SD}(n=3)$. NS, not significant; ${ }^{*} P<0.05 ;{ }^{* *} P<0.01 ;{ }^{* * *} P<0.001$; compared with the 0 time control according to Dunnett's multiple comparisons test.

3.7. Antioxidant Butylated Hydroxyanisole (BHA) Abrogates DK1913-Induced Activation of the Caspase Cascade. Besides BAX-dependent channel formation, loss of mitochondria membrane potential $(\Delta \Psi \mathrm{m})$ contributes to the release of Cyt $c$ as well as that of other mitochondrial apoptogenic factors, such as apoptosis-inducing factor (AIF) and Smac/Diablo [38]. To further evaluate whether DK1913 alters the $\Delta \Psi \mathrm{m}$, we used a membrane potentialsensitive fluorochrome, JC-1, which emits red fluorescence in intact mitochondria with high $\Delta \Psi \mathrm{m}$; in contrast, it emits green fluorescence upon loss of $\Delta \Psi \mathrm{m}$. As shown in Figure 7(a), DK1913 treatment shifted the emission from red to green fluorescence, indicating the loss of $\Delta \Psi m$ in DK1913-induced apoptosis. It has been reported that ROS generation is associated with loss of $\Delta \Psi \mathrm{m}$ and mitochondria-mediated apoptosis [39]. To determine the causal role of ROS generation in DK1913-mediated apoptosis, we tested the effect of butylated hydroxyanisole (BHA), a synthetic antioxidant, on DK1913-mediated loss of $\Delta \Psi \mathrm{m}$. Pretreatment with BHA switched the green fluorescence induced by DK1913 to red fluorescence (Figure 7(a)). To further clarify the role of ROS in DK1913-induced activation of the caspase cascade, we tested the effect of BHA on DK1913-induced caspase activation by immunoblotting. We found that the DK1913 treatment-induced cleavage of caspase-7, caspase-3, and PARP was decreased in the presence of BHA (Figure $7(\mathrm{~b})$ ). These results suggest that DK1913-induced elevation in ROS levels is closely associated with the loss of $\Delta \Psi \mathrm{m}$ of the mitochondria, which in turn facilitates the activation of the mitochondrial caspase cascade.

In this study, we have ascertained the effect of aurora kinase inhibitor on ROS-induced apoptosis in HCT116 colon cancer cells. Similar findings were observed in previous studies demonstrating that Aurora-A inhibitor TCS10 [15] and Aurora-B inhibitor barasertib [40] trigger apoptotic cell death through the excess generation of ROS. Currently, it remains unclear how the aurora kinases inhibition by DK1913 can lead to ROS generation. One possible explanation is that mitotic arrest induced by aurora kinase inhibition could promote oxidative metabolism, increasing intracellular ROS levels. This hypothesis is supported by the previous observation that ROS levels are elevated in mitotic cancer cells [9] and that 


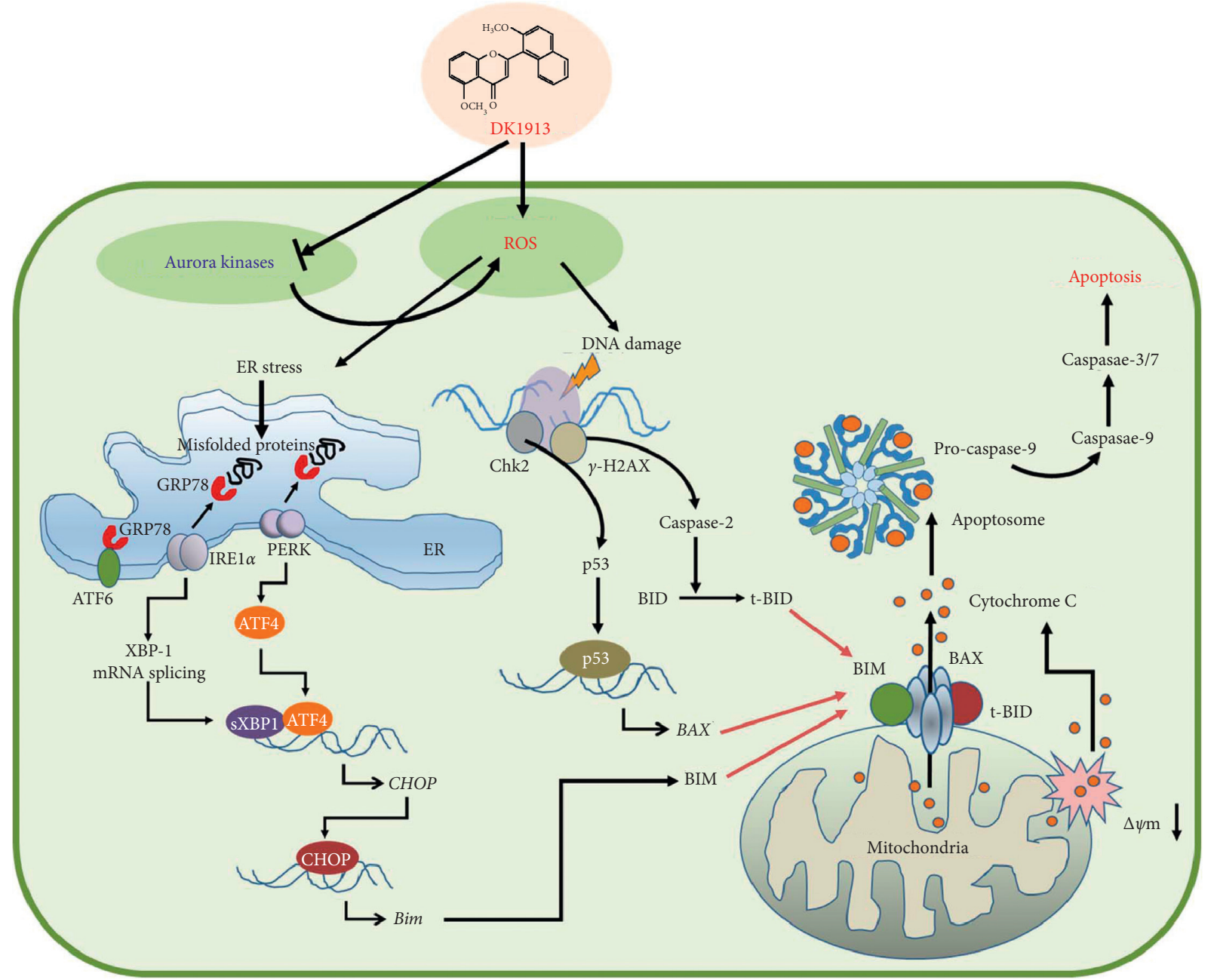

FIgURE 8: Hypothetical model for DK1913-induced apoptosis. Inhibition of aurora kinases by DK1913 induces the generation of intracellular ROS. Aberrant ROS levels activate the UPR pathway of the ER, leading to the accumulation of transcription factor $\mathrm{CHOP}$, which in turn upregulates the proapoptotic BIM. DK1913-induced genotoxic response induces p53-dependent BAX expression and caspase-2-mediated activation of proapoptotic $\mathrm{t}$-BID. Loss of mitochondrial membrane potential $(\triangle \Psi \mathrm{m})$ along with the accumulation of BAX and BH3-only proteins (BIM and t-BID) facilitates Cyt c release from the mitochondria, which leads to the activation of caspase- 9 and caspase-3/7, triggering apoptosis.

accumulated cyclinB/Cdk, a cell cycle regulator involved in driving mitotic entry and progression, during mitotic arrest enhances mitochondrial oxidative metabolism [41]. Indeed, microtubule stabilizer paclitaxel, an antimitotic drug resulting in mitotic arrest, causes oxidative stress [42]. Additional studies are needed to provide mechanistic insight into the mode of action of DK1913 in the generation of ROS.

\section{Conclusion}

The synthetic Aurora kinase inhibitor, DK1913, triggers apoptosis through a ROS-mediated mitochondrial caspase pathway activated via genotoxic and ER stress responses in HCT116 colon cancer cells (Figure 8). As most cancer cells are more sensitive to ROS than normal cells, ROS-producing DK1913 could be used for the selective targeting of cancer cells [43-46]. Further studies are needed to evaluate the in vivo anticancer efficacy of DK1913.

\section{Data Availability}

The data used to support the findings of this study are included within the article and are available from the corresponding author upon request.

\section{Conflicts of Interest}

The authors declare that there are no conflicts of interest regarding the publication of this paper.

\section{Acknowledgments}

This paper was supported by the KU Research Professor Program of Konkuk University and by the Basic Science Research Program through the National Research Foundation of Korea (NRF) funded by the Ministry of Science, ICT, and Future Planning (Grant no. NRF2018R1A2B2004653), Republic of Korea. 


\section{References}

[1] E. A. Nigg, "Mitotic kinases as regulators of cell division and its checkpoints," Nature Reviews Molecular Cell Biology, vol. 2, no. 1, pp. 21-32, 2001.

[2] G. Vader and S. M. Lens, "The aurora kinase family in cell division and cancer," Biochimica et Biophysica Acta, vol. 1786, no. 1 , pp. 60-72, 2008.

[3] A. R. Barr and F. Gergely, "Aurora-A: the maker and breaker of spindle poles," Journal of Cell Science, vol. 120, no. 17, pp. 2987-2996, 2007.

[4] M. Kimura, Y. Matsuda, T. Yoshioka, and Y. Okano, "Cell cycle-dependent expression and centrosome localization of a third human aurora/ipll-related protein kinase, AIK3," Journal of Biological Chemistry, vol. 274, no. 11, pp. 73347340, 1999.

[5] J. R. Bischoff, L. Anderson, Y. Zhu et al., "A homologue of drosophila aurora kinase is oncogenic and amplified in human colorectal cancers," The EMBO Journal, vol. 17, no. 11, pp. 3052-3065, 1998.

[6] J. Fu, M. Bian, Q. Jiang, and C. Zhang, "Roles of aurora kinases in mitosis and tumorigenesis," Molecular Cancer Research, vol. 5, no. 1, pp. 1-10, 2007.

[7] A. O. Walter, W. Seghezzi, W. Korver, J. Sheung, and E. Lees, "The mitotic serine/threonine kinase aurora2/AIK is regulated by phosphorylation and degradation," Oncogene, vol. 19, no. 42, pp. 4906-4916, 2000.

[8] S. Y. Shin, Y. Lee, B. S. Kim et al., "Inhibitory effect of synthetic flavone derivatives on pan-aurora kinases: induction of G2/M cell-cycle arrest and apoptosis in HCT116 human colon cancer cells," International Journal of Molecular Sciences, vol. 19, no. 12, 2018.

[9] J. C. Patterson, B. A. Joughin, B. Van De Kooij, D. C. Lim, D. A. Lauffenburger, and M. B. Yaffe, "ROS and oxidative stress are elevated in mitosis during asynchronous cell cycle progression and are exacerbated by mitotic arrest," Cell Systems, vol. 8, no. 2, pp. 163-167, 2019.

[10] B. Rigas and Y. Sun, "Induction of oxidative stress as a mechanism of action of chemopreventive agents against cancer," British Journal of Cancer, vol. 98, no. 7, pp. 11571160, 2008.

[11] P. T. Schumacker, "Reactive oxygen species in cancer cells: live by the sword, die by the sword," Cancer Cell, vol. 10, no. 3, pp. 175-176, 2006.

[12] J. Wu and R. J. Kaufman, "From acute ER stress to physiological roles of the unfolded protein response," Cell Death \& Differentiation, vol. 13, no. 3, pp. 374-384, 2006.

[13] E. Szegezdi, S. E. Logue, A. M. Gorman, and A. Samali, "Mediators of endoplasmic reticulum stress-induced apoptosis," EMBO Reports, vol. 7, no. 9, pp. 880-885, 2006.

[14] Y. Lee, B. S. Kim, S. Ahn et al., "Anticancer and structureactivity relationship evaluation of 3-(naphthalen-2-yl)N,5-diphenyl-pyrazoline-1-carbothioamide analogs of chalcone," Bioorganic Chemistry, vol. 68, pp. 166-176, 2016.

[15] D. H. Lee, C. G. Kim, Y. Lim, and S. Y. Shin, "Aurora kinase a inhibitor TCS7010 demonstrates pro-apoptotic effect through the unfolded protein response pathway in HCT116 colon cancer cells," Oncology Letters, vol. 14, no. 6, pp. 6571-6577, 2017.

[16] S. Y. Shin, S. Ahn, H. Yoon et al., "Colorectal anticancer activities of polymethoxylated 3-naphthyl-5-phenylpyrazoline-carbothioamides," Bioorganic \& Medicinal Chemistry Letters, vol. 26, no. 17, pp. 4301-4309, 2016.
[17] H.-N. Gil, D. Koh, Y. Lim, Y. H. Lee, and S. Y. Shin, "The synthetic chalcone derivative 2-hydroxy-3' $, 5,5^{\prime}$-trimethoxychalcone induces unfolded protein response-mediated apoptosis in A549 lung cancer cells," Bioorganic \& Medicinal Chemistry Letters, vol. 28, no. 17, pp. 2969-2975, 2018.

[18] J. J. Lum, D. E. Bauer, M. Kong et al., "Growth factor regulation of autophagy and cell survival in the absence of apoptosis," Cell, vol. 120, no. 2, pp. 237-248, 2005.

[19] A. A. Dar, L. W. Goff, S. Majid, J. Berlin, and W. El-Rifai, "Aurora kinase inhibitors-rising stars in cancer therapeutics?" Molecular Cancer Therapeutics, vol. 9, no. 2, pp. 268278, 2010.

[20] J. P. Li, Y. X. Yang, Q. L. Liu et al., "The investigational aurora kinase a inhibitor alisertib (MLN8237) induces cell cycle G2/ $\mathrm{M}$ arrest, apoptosis, and autophagy via p38 MAPK and Akt/ mTOR signaling pathways in human breast cancer cells," Drug Design, Development and Therapy, vol. 9, pp. 1627-1652, 2015.

[21] J. Gaspar, A. Rodrigues, A. Laires et al., "On the mechanisms of genotoxicity and metabolism of quercetin," Mutagenesis, vol. 9, no. 5, pp. 445-449, 1994.

[22] H. Luo, A. Yang, B. A. Schulte, M. J. Wargovich, and G. Y. Wang, "Resveratrol induces premature senescence in lung cancer cells via ROS-mediated DNA damage," PLoS One, vol. 8, no. 3, Article ID e60065, 2013.

[23] J. Boelens, S. Lust, F. Offner, M. E. Bracke, and B. W. Vanhoecke, "Review: the endoplasmic reticulum: a target for new anticancer drugs," In Vivo, vol. 21, no. 2, pp. 215-226, 2007.

[24] L. Zhang, M. B. Hapon, A. A. Goyeneche et al., "Mifepristone increases mRNA translation rate, triggers the unfolded protein response, increases autophagic flux, and kills ovarian cancer cells in combination with proteasome or lysosome inhibitors," Molecular Oncology, vol. 10, no. 7, pp. 1099-1117, 2016.

[25] D. H. Lee, Y. Jung Jung, D. Koh, Y. Lim, Y. H. Lee, and S. Y. Shin, "A synthetic chalcone, 2'-hydroxy-2,3,5'-trimethoxychalcone triggers unfolded protein response-mediated apoptosis in breast cancer cells," Cancer Letters, vol. 372, no. 1, pp. 1-9, 2016.

[26] H. Zinszner, M. Kuroda, X. Wang et al., "CHOP is implicated in programmed cell death in response to impaired function of the endoplasmic reticulum," Genes \& Development, vol. 12, no. 7, pp. 982-995, 1998.

[27] S. Oyadomari and M. Mori, "Roles of CHOP/GADD153 in endoplasmic reticulum stress," Cell Death \& Differentiation, vol. 11, no. 4, pp. 381-389, 2004.

[28] T. Miyashita, S. Krajewski, M. Krajewska et al., "Tumor suppressor p53 is a regulator of bcl-2 and bax gene expression in vitro and in vivo," Oncogene, vol. 9, no. 6, pp. 1799-1805, 1994.

[29] T. Miyashita and J. C. Reed, "Tumor suppressor p53 is a direct transcriptional activator of the human bax gene," Cell, vol. 80, no. 2, pp. 293-299, 1995.

[30] E. P. Rogakou, D. R. Pilch, A. H. Orr, V. S. Ivanova, and W. M. Bonner, "DNA double-stranded breaks induce histone H2AX phosphorylation on serine 139," Journal of Biological Chemistry, vol. 273, no. 10, pp. 5858-5868, 1998.

[31] P. Lassus, X. Opitz-Araya, and Y. Lazebnik, "Requirement for caspase- 2 in stress-induced apoptosis before mitochondrial permeabilization," Science, vol. 297, no. 5585, pp. 1352-1354, 2002.

[32] B. Zhivotovsky and S. Orrenius, "Caspase-2 function in response to DNA damage," Biochemical and Biophysical Research Communications, vol. 331, no. 3, pp. 859-867, 2005. 
[33] N. N. Danial and S. J. Korsmeyer, "Cell death," Cell, vol. 116, no. 2, pp. 205-219, 2004.

[34] R. J. Youle and A. Strasser, "The BCL-2 protein family: opposing activities that mediate cell death," Nature Reviews Molecular Cell Biology, vol. 9, no. 1, pp. 47-59, 2008.

[35] D. C. S. Huang and A. Strasser, "BH3-only proteins-essential initiators of apoptotic cell death," Cell, vol. 103, no. 6, pp. 839-842, 2000.

[36] H. Zou, Y. Li, X. Liu, and X. Wang, "An APAF1 .cytochromecmultimeric complex is a functional apoptosome that activates procaspase-9," Journal of Biological Chemistry, vol. 274, no. 17, pp. 11549-11556, 1999.

[37] Y. A. Lazebnik, S. H. Kaufmann, S. Desnoyers, G. G. Poirier, and W. C. Earnshaw, "Cleavage of poly (ADP-ribose) polymerase by a proteinase with properties like ice," Nature, vol. 371, no. 6495, pp. 346-347, 1994.

[38] G. Kroemer and J. C. Reed, "Mitochondrial control of cell death," Nature Medicine, vol. 6, no. 5, pp. 513-519, 2000.

[39] T. Satoh, Y. Enokido, H. Aoshima, Y. Uchiyama, and H. Hatanaka, "Changes in mitochondrial membrane potential during oxidative stress-induced apoptosis in PC12 cells," Journal of Neuroscience Research, vol. 50, no. 3, pp. 413-420, 1997.

[40] A. Zekri, Y. Mesbahi, S. Ghanizadeh-Vesali, K. Alimoghaddam, A. Ghavamzadeh, and S. H. Ghaffari, "Reactive oxygen species generation and increase in mitochondrial copy number," Anti-Cancer Drugs, vol. 28, no. 8, pp. 841-851, 2017.

[41] Z. Wang, M. Fan, D. Candas et al., "Cyclin B1/Cdk1 coordinates mitochondrial respiration for cell-cycle G2/M progression," Developmental Cell, vol. 29, no. 2, pp. 217-232, 2014.

[42] J. Alexandre, Y. Hu, W. Lu, H. Pelicano, and P. Huang, "Novel action of paclitaxel against cancer cells: bystander effect mediated by reactive oxygen species," Cancer Research, vol. 67, no. 8, pp. 3512-3517, 2007.

[43] L. Raj, T. Ide, A. U. Gurkar et al., "Selective killing of cancer cells by a small molecule targeting the stress response to ROS," Nature, vol. 475, no. 7355, pp. 231-234, 2011.

[44] D. J. Adams, M. Dai, G. Pellegrino et al., "Synthesis, cellular evaluation, and mechanism of action of piperlongumine analogs," Proceedings of the National Academy of Sciences, vol. 109, no. 38, pp. 15115-15120, 2012.

[45] X. Cui, "Reactive oxygen species: the achilles' heel of cancer cells?” Antioxidants \& Redox Signaling, vol. 16, no. 11, pp. 1212-1214, 2012.

[46] S. Y. Shin, J. M. Lee, M. S. Lee et al., "Targeting cancer cells via the reactive oxygen species-mediated unfolded protein response with a novel synthetic polyphenol conjugate," Clinical Cancer Research, vol. 20, no. 16, pp. 4302-4313, 2014. 